\title{
Optimization-Driven Design of Compact UWB MIMO Antenna
}

\author{
Adrian Bekasiewicz ${ }^{1}$, Slawomir Koziel ${ }^{1}$, and Tom Dhaene ${ }^{2}$ \\ ${ }^{1}$ Engineering Optimization \& Modeling Center, Reykjavik University, Reykjavik, Iceland \\ ${ }^{2}$ Department of Information Technology, Ghent University, Ghent, Belgium
}

\begin{abstract}
In the paper, a concept and design of a compact MIMO antenna is presented. In our approach, an improved space filling is obtained by constraining an individual antenna layout and optimizing it for minimum size. The MIMO design is constructed by composing the two of previously designed structures. Because the design problem is heavily constrained both in terms of geometry and electrical performance objectives, surrogate-based optimization techniques are used to accomplish the design process in reasonable timeframe. The final MIMO design features a small size of only $565 \mathrm{~mm}^{2}$ and exhibits good isolation in the entire UWB band.
\end{abstract}

Index Terms-UWB antennas, MIMO antennas, computeraided design, EM-driven design, topology optimization, surrogate-based optimization.

\section{INTRODUCTION}

Design of compact ultra-wideband (UWB) antennas for space-limited [1] and wearable applications [2] is an important topic of research in antenna engineering. From the UWB system standpoint, appropriate design of the antenna structure can lead to considerable complexity reduction of the RF frontend. One of the important challenges of UWB antenna design is prevention of multipath fading of the signals. This problem can be addressed by means of multiple-inputmultiple-output (MIMO) technology where a single antenna is replaced by a suitable multi-radiator design which may prevent fading by appropriate post processing of the signals [3].

Despite of the advantages of the MIMO structures, their utilization for space-limited applications is problematic: allocation of radiators in close proximity of each other leads to the increased mutual couplings and, consequently, degradation of the antenna performance in terms of isolation. Nevertheless, several implementations of small MIMO antennas have been reported in literature. The most common realizations are based on orthogonally allocated antennas with the common ground plane and additional modifications to increase isolation between the components [4]. However, the structures with parallel radiators have been also reported [5]. Alternative approaches to achieve compact design include utilization of multi-fed radiator [6], stripline-fed designs in back-to-back configuration [7], as well as protruded ground plane realizations [3]. External dimensions of aforementioned designs vary from $48 \mathrm{~mm} \times 48$ $\mathrm{mm}$ [8] to even $29 \mathrm{~mm} \times 27 \mathrm{~mm} \mathrm{[3]}$.

A practical design issue is that compact MIMO antennas are characterized by complex multi-parameter geometries so that their reliable evaluation can only be obtained by means of highfidelity EM simulations [3], [4]. At the same time, utilization of conventional experience-driven strategies for manual adjustment of geometry parameters (parameter sweeps, typically one parameter at a time) is hindered due to complex relations between the antenna dimensions and responses. Truly optimum designs can only be obtained using rigorous numerical optimization. Unfortunately, conventional optimization methods are computationally expensive. For example, gradientbased optimization with numerical derivatives takes up to a few hundred of EM analyses to converge even if the number of adjustable parameters is smaller than ten [10].

The state of the art analysis indicates that considerable reduction of the simulation-driven design effort can be obtained either through gradient search with adjoint sensitivities [11], [12], or by employing fast surrogate models [13], [14]. The first option is conceptually simpler and with strong theoretical foundations, although commercial availability of adjoint technology in EM solvers is still very limited. Surrogate-based optimization (SBO) [13], [14] seems to be more versatile although more difficult in implementation and automation [15]. Popular SBO methods applied for antenna design include several types of space mapping [14], [16] also in combination with response surface approximation models [17], as well as various response correction techniques (SPRP [18], manifold mapping [19]), and, recently, feature-based optimization [20].

In this work, we propose a new concept of compact MIMO antenna and describe its efficient design optimization procedure. The concept is based on more efficient utilization of available space by constraining an individual antenna layout with further composition of such antennas into a MIMO structure. The design process is carried out in two stages. At the first stage, UWB operation of single radiator is obtained. Expedited design of the antenna is achieved by exploiting surrogate-assisted design techniques. The optimization process aims at reduction of the antenna size while maintaining its acceptable electrical performance, which is controlled using a penalty function approach. At the second stage, the optimized antenna is utilized to construct a MIMO structure. The final adjustment aimed at ensuring desired levels of isolation between radiators is controlled using a separate set of parameters. The footprint of the final design is only $565 \mathrm{~mm}^{2}$ and the antenna outperforms comparable structures reported in the literature in terms of the size, while ensuring competitive isolation characteristics. 


\section{Proposed MIMO ANTENNA STRUCTURE}

Consider a UWB monopole antenna shown in Fig. 1. The structure is based on a design of [4] and it consists of a rectangular-shape radiator fed through a microstrip line and a modified ground plane with a rectangle slot and stub. Both are introduced to enhance antenna electrical performance, while ensuring small dimensions. The structure is described by eleven parameters $\boldsymbol{x}_{a}=\left[\begin{array}{lllllllllll}l_{g} & g & a_{1} & a_{2} & l_{1} & l_{2} & w_{1} & s_{1} & s_{2} & o_{1} & o_{3}\end{array}\right]^{T}$. The parameter $o_{2}=0.5 \cdot a_{2}$, whereas $w_{0}=1.7$ remains fixed in order to ensure $50 \Omega$ input impedance. The structure is implemented on a Taconic RF-35 substrate $\left(\varepsilon_{r}=3.5, \tan \delta=0.0018, \mathrm{~h}=\right.$ $0.762 \mathrm{~mm})$. The high-fidelity model $\boldsymbol{R}_{f}$ of the antenna is implemented in CST Microwave Studio and evaluated using its time domain solver [21]. The design is electrically small and the EM model is supplemented with the SMA connector to ensure reliable simulation results (see Fig. 1(b)). The model consists of $\sim 6,000,000$ hexahedral mesh cells and its average simulation time on a dual Xeon E5540 machine with 6 GB $\mathrm{RAM}$ is $55 \mathrm{~min}$. In the design process, we also utilize an auxiliary coarse-discretization model $\boldsymbol{R}_{c}$ which contains $\sim 100,000$ cells; its simulation time is $60 \mathrm{~s}$.

The antenna of Fig. 1 can be arranged in multi-radiator configuration to construct fairly compact MIMO antenna [4]. Unfortunately, ensuring acceptable isolation between radiators utilized in geometrically restricted MIMO structures is often achieved at a cost of complex and multi-parameter geometries. Therefore, their simulation-driven design using numerical optimization is difficult and computationally expensive.

These challenges can be addressed by a two-stage design procedure as follows:

1. Design of a radiator and optimization of its geometrical parameters $\boldsymbol{x}_{a}$ to ensure compact dimensions and acceptable electrical performance within a UWB band;

2. Construction of a MIMO antenna using two orthogonally allocated radiators interconnected through a common ground plane; adjustment of the MIMO-related parameters $\boldsymbol{x}_{b}$ to obtain desired isolation between the radiators.

This decomposition of the design process is possible because interconnection between the radiators has minor influence on their reflection properties. At the same time, separating the radiator and the MIMO antenna parameters reduces complexity of the problem optimization problem.

The MIMO structure composed of two orthogonally allocated antennas (see Fig. 1) is illustrated in Fig. 2. The MIMO-related parameters are $\boldsymbol{x}_{b}=\left[l_{c} w_{c}\right]^{T}$. The high-fidelity model $\boldsymbol{R}_{f}^{*}$ consist of $\sim 10,500,000$ cells and its average simulation time is $3 \mathrm{~h}$.

Two design objectives are considered in the first stage of the design process: (i) minimization of antenna footprint and (ii) maintaining the reflection $\left|S_{11}\right|$ below the acceptable threshold level $S_{\max }$. The antenna footprint $S(\boldsymbol{x})$ is defined as $w_{m} \times l_{m}$ rectangle where $w_{m}=o_{1}+o_{2}+o_{3}$ and $l_{m}=l_{g}+l_{1}+w_{1}$. The aim of the second stage is to maximize isolation between radiators.

\section{SURROGATE-ASSISTED ANTENNA DESIGN}

The design process aims at optimizing the high-fidelity antenna model $\boldsymbol{R}_{f}$ with respect to the two objectives listed in the previous section. It is formulated as

$$
\boldsymbol{x}^{*}=\arg \min _{\boldsymbol{x}} U\left(\boldsymbol{R}_{f}(\boldsymbol{x})\right)
$$

where $U$ is scalar merit function, whereas $\boldsymbol{x}^{*}$ is the optimum design to be found. Size reduction is selected to be the primary goal, whereas acceptable electrical performance of the antenna will be enforced by means of a penalty function approach. Thus, the function $U$ is defined as

$$
U\left(\boldsymbol{R}_{f}(\boldsymbol{x})\right)=S(\boldsymbol{x})+\beta \cdot c\left(\boldsymbol{R}_{f}(\boldsymbol{x})\right)^{2}
$$

where $S(\boldsymbol{x})$ denotes the antenna footprint, $\beta$ is a penalty factor (here, $\beta=1000$ ), and $c$ is a penalty function defined as

$$
c\left(\boldsymbol{R}_{f}(\boldsymbol{x})\right)=\max \left\{\left(\max \left\{\left|S_{11}\right|_{3.1 \mathrm{GHz}} \text { to } 10.6 \mathrm{GHz}\right\}+S_{\max }\right) / S_{\max }, 0\right\}
$$

The penalty term gives a non-zero contribution to (2) if the maximum (worst-case) of in-band reflection is above $S_{\max }$.

Due to high cost of evaluating the high-fidelity model, direct solving of (1) is impractical. Here, to facilitate the design we use a surrogate-based optimization (SBO) process that generates a series $\boldsymbol{x}^{(i)}, i=0,1, \ldots$, of approximate solution to (1) as follows

$$
\boldsymbol{x}^{(i+1)}=\arg \min _{\boldsymbol{x}} U\left(\boldsymbol{R}_{s}^{(i)}(\boldsymbol{x})\right)
$$

$\boldsymbol{R}_{s}^{(i)}$ is a surrogate model at iteration $i$. The surrogate is obtained by suitable correction (here, output space mapping [14]) of a local response surface approximation (RSA) model [13] constructed in the vicinity of the current design $\boldsymbol{x}^{(i)}$. The latter is a second-order polynomial model (without mixed terms) generated using $2 n+1 \boldsymbol{R}_{c}$ samples ( $n$ being the number of adjustable parameters) allocated using star-distribution scheme [14]. The low-fidelity model setup is briefly described in Section II, whereas more detailed discussion on the local RSA models can be found in [13], [14].

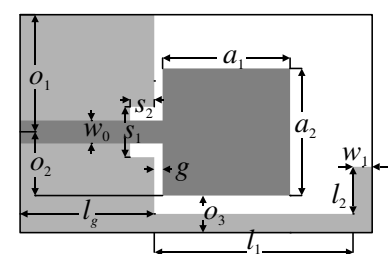

(a)

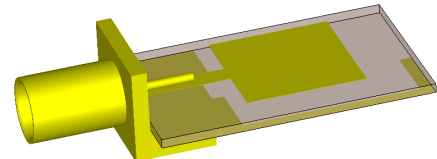

(b)
Fig. 1. Compact UWB monopole: (a) geometrical details of the radiator with highlighted dimensions $\boldsymbol{x}_{a}$ (the ground plane is marked using light-shade gray); (b) 3D visualization of the antenna with the SMA connector.

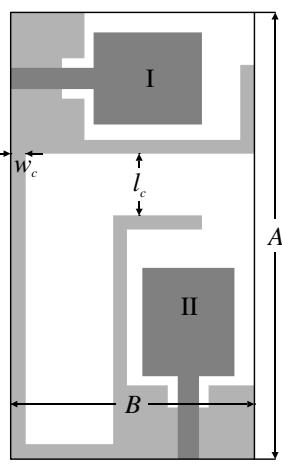

(a)

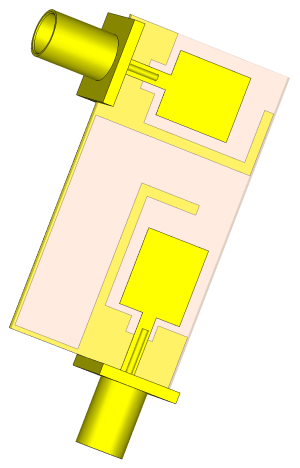

(b)
Fig. 1. Compact MIMO antenna: (a) geometry of the MIMO structure composed of the two orthogonal radiators (here denoted as I and II, respectively) with highlighted parameters $\boldsymbol{x}_{b}$ (the ground plane is marked using light-shade gray); and (b) 3D visualization of the MIMO antenna. 


\section{Simulation Results}

The antenna structure of Fig. 1 has been optimized using procedure of Section III. The initial is $\boldsymbol{x}_{a}{ }^{0}=\left[\begin{array}{lll}7.0 & 1.010 .010 .0\end{array}\right.$

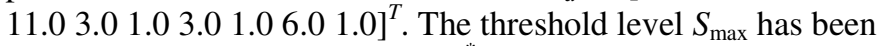
set to $-12 \mathrm{~dB}$. The final design $\boldsymbol{x}_{a}{ }^{*}=\left[\begin{array}{l}5.97 \\ 0.74\end{array} 8.777 .4612 .61\right.$ $\begin{array}{llllll}6.05 & 1.11 & 3.3 & 1.8 & 6.1 & 1.61]^{T} \text { has been obtained after only seven }\end{array}$ iterations of (4). The reflection characteristics of the initial and the final design are compared in Fig. 2. The optimized structure features fairly compact dimensions of $11.5 \mathrm{~mm} \times$ $19.7 \mathrm{~mm}$ and overall footprint of $227 \mathrm{~mm}^{2}$. It should be noted that optimized design fulfills the imposed electrical specification $\left(\left|S_{11}\right| \leq-12 \mathrm{~dB}\right.$ in $3.1 \mathrm{GHz}$ to $\left.10.6 \mathrm{GHz}\right)$. This gives sufficient margin in case of degrading antenna reflection upon assembling the MIMO antenna and adjustment of the MIMO parameters $\boldsymbol{x}_{b}$. The overall optimization cost of the radiator corresponds to about $11 \boldsymbol{R}_{f}$ model simulations.

In the next stage, the MIMO antenna has been constructed using two orthogonally allocated radiators, both parameterized using $\boldsymbol{x}_{a}$. The response characteristics of MIMO for $\boldsymbol{x}_{b}=[9$ $0.5]^{T}$ are shown in Fig. 3. The obtained design is characterized by dimensions of $28.7 \mathrm{~mm} \times 19.7 \mathrm{~mm}$ and footprint of only $565 \mathrm{~mm}^{2}$. It should be emphasized that computational cost of $\boldsymbol{R}_{f}{ }^{*}$ model evaluation is significant, and thus the influence of $\boldsymbol{x}_{b}$ on behavior of the structure has been determined through parametric studies. The results shown in Fig. 4 indicate that influence of $w_{c}$ on isolation between the radiators is limited, whereas the increase of $l_{c}$ noticeably improves isolation at the lower frequency range.

The reduced isolation of the MIMO antenna for lower frequencies can be explained using the surface current distributions shown in Fig. 5. It can be observed that for both radiators, the current density on the ground plane stubs is high at $3.1 \mathrm{GHz}$ (see Fig. 5(a)-(b)), which implies that they are of high importance for low-frequency operation of both radiators. However, the role of stubs is also to work as parasitic elements that reduce radiator coupling [4]. For higher frequencies (e.g., $6 \mathrm{GHz}$ and $9 \mathrm{GHz}$ as illustrated in Figs. 5(c)-(f)) the proposed MIMO configuration ensures very good isolation.

The discussed design has been compared with other stateof-the-art MIMO antennas [3], [4], [9] in terms of the occupied area and in-band isolation. To account for the differences between the substrate parameters of the compared antennas, their dimensions have been expressed in terms of guided wavelength $\lambda_{g}$ (defined for the $50 \mathrm{Ohm}$ line operating at 6.85 $\mathrm{GHz}$ center frequency). The results collected in Table I indicate that the proposed antenna outperforms other designs in terms of the size, while ensuring competitive isolation.

\section{TABLE I. COMPARISON OF COMPACT MIMO ANTENNAS}

\begin{tabular}{ccccc}
\hline \hline Antenna & $\begin{array}{c}\text { Dimensions } \\
\mathrm{mm} \times \mathrm{mm}\end{array}$ & $\begin{array}{c}\text { Effective } \\
\lambda_{\mathrm{g}} \times \lambda_{\mathrm{g}}\end{array}$ & $\begin{array}{c}\text { Footprint }^{\#} \\
\lambda_{\mathrm{g}}{ }^{2}\end{array}$ & $\begin{array}{c}\text { In-band } \\
\text { isolation [dB] }\end{array}$ \\
\hline Design [9] & $32.0 \times 32.0$ & $1.34 \times 1.34$ & 1.78 & 15.0 \\
Design [4] & $40.0 \times 26.0$ & $1.53 \times 0.99$ & 1.52 & 11.5 \\
Design [3] & $29.0 \times 27.0$ & $1.21 \times 1.13$ & 1.37 & 11.0 \\
This work & $28.7 \times 19.7$ & $1.10 \times 0.75$ & 0.83 & 14.5 \\
\hline \hline
\end{tabular}

"For fair comparison, the antenna size is expressed in terms of the guided wavelength corresponding to the substrate properties the design is implemented on.

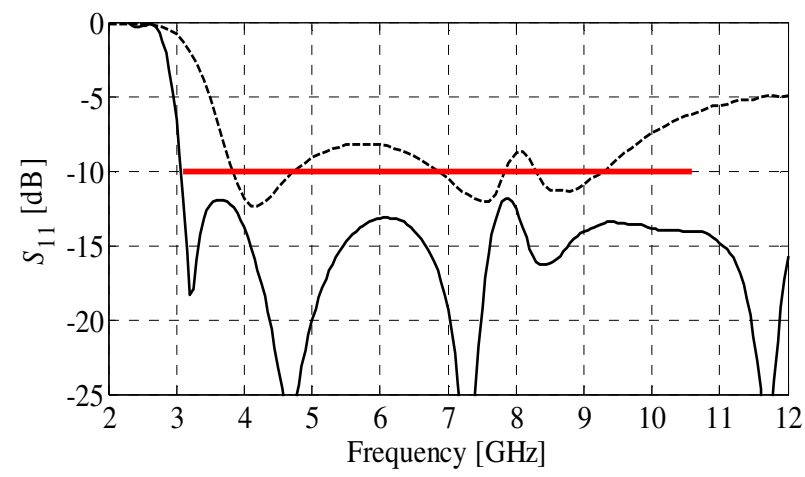

Fig. 2. Reflection characteristics of the monopole antenna at the initial (- - ) and the final design (- $)$.

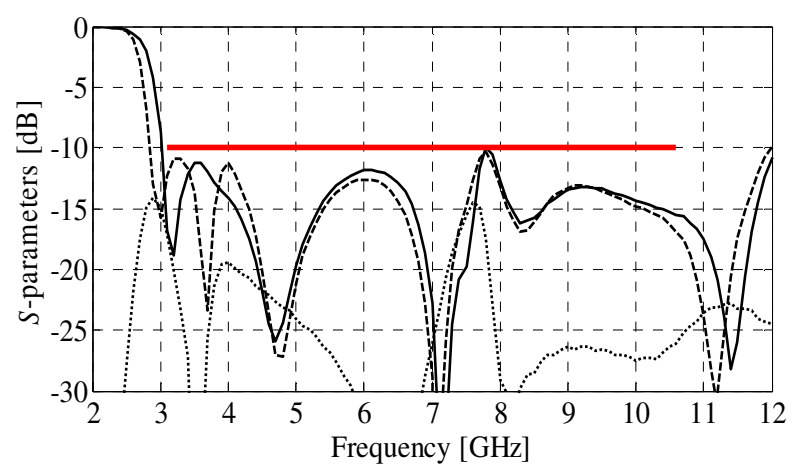

Fig. 3. S-parameter characteristics of the compact MIMO antenna. Solid and dashed lines denote reflections of the radiator I and II (see Fig. 1(c)), respectively, whereas isolation is marked with a dotted line.

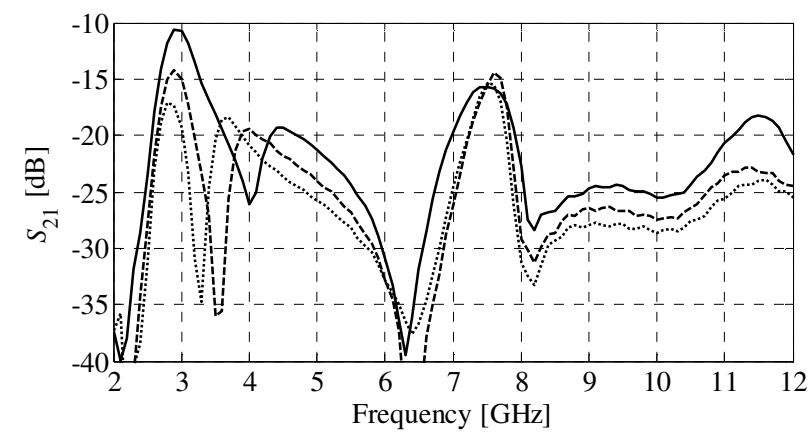

(a)

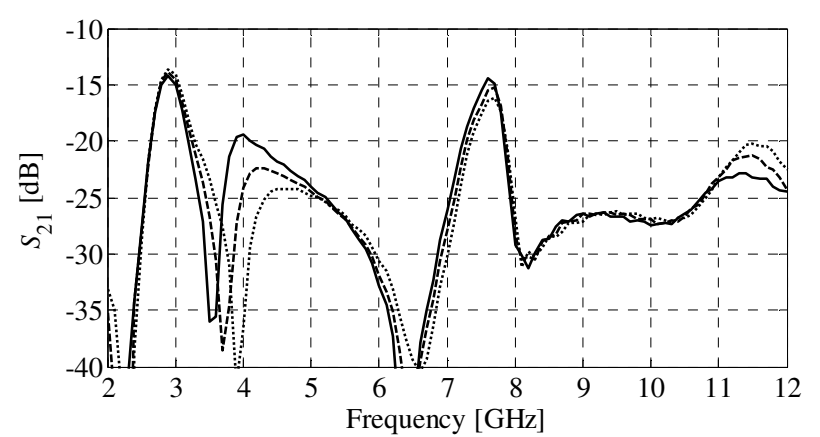

(b)

Fig. 4. Parametric study of the MIMO antenna: (a) isolation for $w_{c}=0.5 \mathrm{~mm}$ and $l_{c}$ of $5 \mathrm{~mm} \mathrm{(-),} 9 \mathrm{~mm} \mathrm{(--)}$ and $12 \mathrm{~mm} \mathrm{( \cdots )}$; (b) isolation for $l_{c}=9 \mathrm{~mm}$

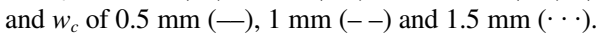




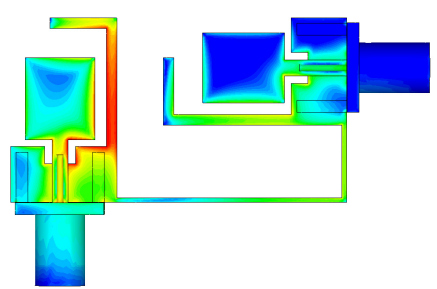

(a)

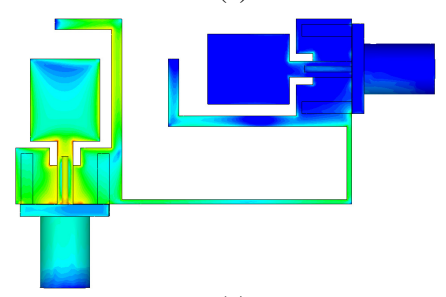

(c)

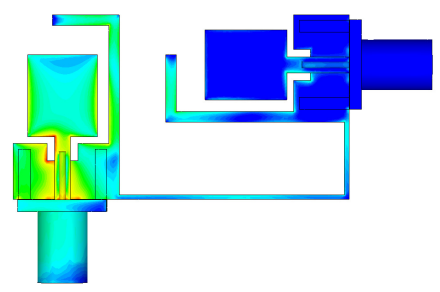

(e)

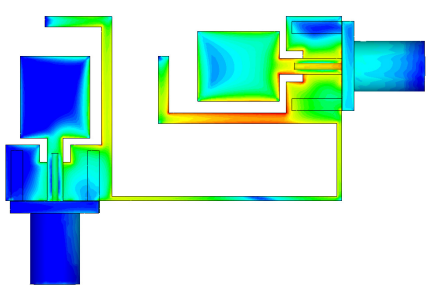

(b)

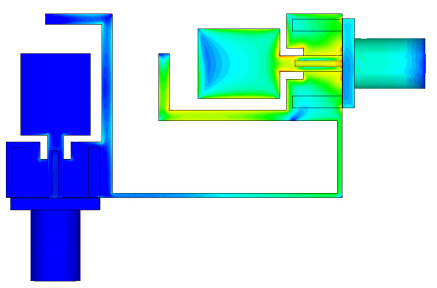

(d)

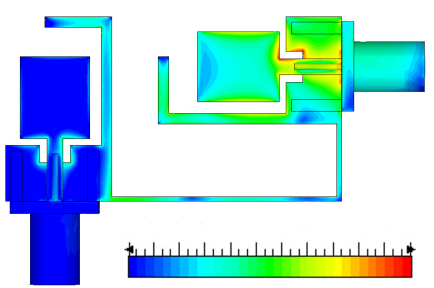

(f)
Fig. 5. Surface current distribution of the proposed compact MIMO antenna (scale: 0 to 40; unit: $\mathrm{dB} 1 \mathrm{~A} / \mathrm{m}$ ) at: (a)-(b) $3.1 \mathrm{GHz}$; (c)-(d) $6 \mathrm{GHz}$; (e)-(f) 9 $\mathrm{GHz}$. Left- and right-hand side distributions are obtained when exciting the radiator I and II, respectively.

\section{CONCLUSION}

A structure and design optimization of a compact UWB MIMO antenna has been discussed. The proposed approach exploits decomposition of the problem into two separate stages, i.e., design of a single radiator and its incorporation into the MIMO structure. At each stage of the process, separate sets of parameters are utilized, so that the final adjustment of the MIMO antenna geometry can be carried out using only two parameters. Variable-fidelity EM antenna models are utilized to expedite the process of geometry parameter optimization. Our approach is demonstrated using a compact MIMO structure consisting of two UWB monopole antennas. The obtained structure features footprint of only $565 \mathrm{~mm}^{2}$. The design outperforms the competitive MIMO structures in terms of the size and exhibits very good electrical performance. Our further work will be focused on application of the introduced methodology to design of other MIMO structures with the focus on improving isolation between radiators as well as increasing their number.

\section{ACKNOWLEDGEMENT}

The authors would like to thank Computer Simulation Technology AG, Darmstadt, Germany, for making CST Microwave Studio available. This work is partially supported by the Icelandic Centre for Research (RANNIS) Grant 141272051 and by National Science Centre of Poland Grant 2014/15/B/ST7/04683.

\section{REFERENCES}

[1] A. Bekasiewicz, S. Koziel, "Structure and Computationally-Efficient Simulation-Driven Design of Compact UWB Monopole Antenna," IEEE Ant. Wireless Prop. Lett., vol. 14, pp. 1282-1285, 2015.

[2] M. Ur-Rehman, Q.H. Abbasi, M. Akram, C. Parini, "Design of bandnotched ultra wideband antenna for indoor and wearable wireless communications," IET Microwaves, Ant. Prop., vol. 9, no. 3, pp. 243$251,2015$.

[3] J.-F. Li, Q.-X. Chu, Z.-H. Li, and X.-X. Xia, "Compact Dual BandNotched UWB MIMO Antenna With High Isolation," IEEE Trans. Ant. Prop., vol. 61, no. 9, pp. 4759-4766, 2013.

[4] L. Liu, S.W. Cheung, and T.I. Yuk, "Compact MIMO Antenna for Portable Devices in UWB Applications," IEEE Trans. Antennas Prop., vol. 61, no. 8, pp. 4257-4264, 2013.

[5] X.-L. Liu, Z.-D. Wang, Y.-Z. Yin, J. Ren, J.-J. Wu, “A Compact Ultrawideband MIMO Antenna Using QSCA for High Isolation," IEEE Ant. Wireless Prop. Lett., vol. 13, pp. 1497-1500, 2014.

[6] C.-X. Mao, Q.-X. Chu, "Compact Coradiator UWB-MIMO Antenna With Dual Polarization," IEEE Trans. Ant. Prop., vol. 62, no. 9, pp. 4474-4480, 2014.

[7] T.K. Roshna, U. Deepak, V.R. Sajitha, K. Vasudevan, P. Mohanan, "A Compact UWB MIMO Antenna With Reflector to Enhance Isolation," IEEE Trans. Ant. Prop., vol. 63, no. 4, pp. 1873-1877, 2015.

[8] P. Gao, S. He, X. Wei, Z. Xu, N. Wang, Y. Zheng, "Compact Printed UWB Diversity Slot Antenna With 5.5-GHz Band-Notched Characteristics," IEEE Ant. Wireless Prop. Lett., vol. 13, pp. 376-379, 2014.

[9] J. Ren, W. Hu, Y. Yin, R. Fan, "Compact Printed MIMO Antenna for UWB Applications," IEEE Ant. Wireless Prop. Lett., vol. 13, pp. 1517$1520,2014$.

[10] S. Koziel and S. Ogurtsov, "Antenna design by simulation-driven optimization. Surrogate-based approach," Springer, 2014.

[11] S. Koziel, and A. Bekasiewicz, "Fast EM -Driven Size Reduction of Antenna Structures By Means of Adjoint Sensitivities and Trust Regions," to appear, IEEE Ant. Wireless Prop. Lett., 2015.

[12] M. Ghassemi, M. Bakr and N. Sangary, "Antenna design exploiting adjoint sensitivity-based geometry evolution," IET Microwaves Ant. Prop., vol. 7, no. 4, pp. 268-276, 2013.

[13] A.I.J. Forrester, and A.J. Keane, "Recent advances in surrogate-based optimization," Progress in Aerospace Sciences, vol. 45, pp. 50-79, 2009.

[14] S. Koziel, X.S. Yang, and Q.J. Zhang (Eds.), "Simulation-driven design optimization and modeling for microwave engineering", Imperial College Press, 2012.

[15] S. Koziel, and S. Ogurtsov, "Computational-budget-driven automated microwave design optimization using variable-fidelity electromagnetic simulations," Int. J. RF \& Microwave CAE, vol. 23, pp. 349-356, 2013.

[16] S. Koziel, J.W. Bandler, and K. Madsen, "A space mapping framework for engineering optimization: theory and implementation," IEEE Trans. Microw. Theory Tech., vol. 54, pp. 3721-3730, 2006.

[17] S. Koziel, A. Bekasiewicz, "Fast Multi-Objective Optimization of Narrow-Band Antennas Using RSA Models and Design Space Reduction," IEEE Ant. Wireless Prop. Lett., vol. 14, pp. 450-453, 2015.

[18] S. Koziel, S. Ogurtsov, and S. Szczepanski, "Rapid antenna design optimization using shape-preserving response prediction," Bulletin of the Polish Academy of Sciences. Tech. Sci., vol. 60, pp. 143-149, 2012.

[19] S. Koziel, L. Leifsson, and S. Ogurtsov, "Reliable EM-driven microwave design optimization using manifold mapping and adjoint sensitivity," Microwave Opt. Tech. Lett., vol. 55, pp. 809-813, 2013.

[20] S. Koziel, and J.W. Bandler, "Rapid Yield Estimation and Optimization of Microwave Structures Exploiting Feature-Based Statistical Analysis," IEEE Trans. Microwave Theory Tech., vol. 63, no. 1, pp. 107-114, 2015.

[21] CST Microwave Studio, ver. 2013, CST AG, Bad Nauheimer Str. 19, D64289 Darmstadt, Germany, 2013. 